\title{
Digestibility Coefficients and Energy in Alfalfa Hay from Chimborazo and Tungurahua
}

\section{Coeficientes de Digestibilidad y Energía útil en Henos de Alfalfa de Chimborazo y Tungurahua}

\author{
P. Guevara, S. López, and P. Andino
}

Carrera de Zootecnia, Carrera de Medicina Veterinaria, Facultad de Ciencias Pecuarias, Escuela Superior Politécnica de Chimborazo, Riobamba, Ecuador

I International Seminar of Livestock and Agroindustrial Production ESPOCH 2020

Corresponding Author:

P. Guevara

hguevara@espoch.edu.ec

Published: 2 September 2021

Production and Hosting by Knowledge E

(c) P. Guevara et al. This article is distributed under the terms of the Creative Commons Attribution License, which permits unrestricted use and redistribution provided that the original author and source are credited.
S OPEN ACCESS

\section{Abstract}

This study was part of a project (Grasas By Pass), funded by Ciencias Pecuarias ESPOCH. Samples from Chimborazo and Tungurahua were analyzed. The statistical analysis included descriptive analysis and significance tests, including Tukey test. The following results of ranges were found in the first phase: humidity: $7.79 \%( \pm 0.06)-9.99 \%( \pm 0.08)$; ashes: $9.16 \%( \pm 0.14)-$ $10.50 \%$ ( \pm 0.01 ); protein: $18.73 \%( \pm 0.10)-21.37 \%( \pm 0.52)$; crude fiber: $31.64 \%( \pm 0.26)-41.69 \%$ ( \pm 0.11); ethereal extract: $1.36 \%( \pm 0.22)-2.13 \%$ ( \pm 0.50$)$; and ELN: $28.30 \%( \pm 0.09)-34.46 \%( \pm$ $0.24)$. The results from the second phase were: F.D.N.: $41.39 \%( \pm 1.32)-51.77 \%( \pm 0.01)$; F.D.A.: $37.36 \%( \pm 0.26)-45.47 \%( \pm 1.04)$; and L.D.A.: $7.85 \%( \pm 0.48)-9.30 \%( \pm 0.39)$. In vivo digestibility tests were developed in metabolic cages with six sheep per treatment for 11 days. The following results were found: Dry matter digestibility coefficient (C.D.M.S.): $60.78 \%-66.42 \%$; organic matter (C.D.M.O): $61.97 \%-68.24 \%$; protein (C.D.P.B.): $74.35 \%-80.23 \%$; crude fiber (C.D.F.C): 43.73\% - 59.39\%; ethereal extract (C.D.E.E.): $26.56 \%$ - 51.76\%; and E.L.N. (C.D.ELN): $67.26 \%-$ 81.96\%. Finally, the Van Es equations were applied to estimate the values of E.M., E.NI., E.Ng. and N.D.T. The digestibility and energy coefficients for the hay from Chimborazo - San Juan were higher, and there were lower values for Tungurahua - Cunchibamba.

Keywords: chemical composition, digestibility coefficient, useful energy.

\section{Resumen}

Como parte del proyecto financiado Grasas By Pass, de la Facultad de Ciencias Pecuarias ESPOCH; se analizaron muestras procedentes de Chimborazo y Tungurahua; el análisis estadístico incluyó análisis descriptivo y pruebas de significancia según Tukey $P<0.0001$, en la primera etapa se realizó el análisis proximal que reportó rangos de: humedad $7,79 \%( \pm 0,06)-$ $9,99 \%( \pm 0,08)$, cenizas $9,16 \%( \pm 0,14)-10,50 \%( \pm 0,01)$, proteína $18,73 \%( \pm 0,10)-21,37 \%( \pm 0,52)$, fibra cruda $31,64 \%( \pm 0,26)-41,69 \%( \pm 0,11)$, extracto etéreo $1,36 \%( \pm 0,22)-2,13 \%( \pm 0,50)$ y E.L.N. 28,30\% $( \pm 0,09)-34,46 \%( \pm 0,24)$. La segunda fase analizó: F.D.N. con valores de $41,39 \%$ $( \pm 1,32)-51,77 \%( \pm 0,01)$, F.D.A. $37,36 \%( \pm 0,26)-45,47 \%( \pm 1,04)$ y L.D.A. $7,85 \%( \pm 0,48)-9,30 \%$ $( \pm 0,39)$. Se desarrolló las pruebas de digestibilidad In vivo en jaulas metabólicas con 6 ovinos por tratamiento durante 11 días, encontrándose rangos para el coeficiente de digestibilidad de la materia seca (C.D.M.S.) desde 60,78\% - 66,42\%; materia orgánica (C.D.M.O.) de 61,97\% a $68,24 \%$, proteína (C.D.P.B.) de $74,35 \%$ a $80,23 \%$, fibra cruda (C.D.F.C) desde $43,73 \%$ hasta $59,39 \%$, extracto etéreo (C.D.E.E.) $26,56 \%$ a 51,76\%, y E.L.N. (C.D.ELN) desde $67,26 \%$ hasta $81,96 \%$. Finalmente se aplicó las ecuaciones de Van Es para estimar los valores de E.M., E.NI., E.Ng. N.D.T. Siendo más elevados los coeficientes de digestibilidad y energía para los henos de Chimborazo - San Juan y valores más bajos para Tungurahua- Cunchibamba. 


\section{Introducción}

La Cordillera de los Andes, presenta zonas situadas a partir de los 1.500 m.s.n.m. hasta los 4.800 m.s.n.m.. En los valles interandinos ubicados entre los 2.600 y 3.000 m.s.n.m. [1], tiene un clima lluvioso y frío de noviembre a abril y seco de mayo a octubre [2]. Las provincia de Chimborazo y Tungurahua pertenecen a la región húmedo temperado RHTE, la altitud varía entre los 1.800 a 3.000 m.s.n.m. [3].

Según el Instituto Nacional de Estadística y Censos del Ecuador [4], la superficie de labor agropecuaria, con patos cultivados en el 2012 fue de 3.357 .167 millones de hectáreas; Tungurahua y Chimborazo con 31.523 y 54.052 Ha respectivamente [4]. Las principales variedades de pastos cultivados para las regiones templadas y frías: Alfalfa, Ray grass, pasto azul, trébol y vicia [1].

La alfalfa es una leguminosa de alto valor nutritivo [5], y por su gran aptitud agronómica. Pestico et al. [6], indican que es un forraje de excelente calidad por su elevado valor proteico y valor energético. Guerrero-rodríguez [7] y Juan et al. [8] manifiestan que el valor de la alfalfa radica en su alto potencial de producción de materia seca, alta concentración de proteína, alta digestibilidad. La alfalfa es uno de los cultivos más valiosos, tanto en pastoreo directo como enlas distintas formas en que su forraje puede ser conservado [8], que es la henificación. Esta consiste en la desecación de los pastos o forrajes en el menor tiempo posible con el sol y el aire, hasta un nivel de $80-85 \%$ de MS, para así reducir la cantidad de agua de sus tallos y hojas. De esta forma el material obtenido se puede almacenar sin que se produzcan fermentaciones ni desarrollo de microorganismos [9, 10]. En el Ecuador, la alfalfa se encuentra en los valles interandinos subtropicales, preferentemente en alturas entre 1500 y 2400 m, pero puede crecer por sobre los $3000 \mathrm{~m}$ en forma marginal [11].

El valor nutritivo se define como la presencia y disponibilidad de nutrientes y puede describirse en términos de concentración

de nutrientes [7] Existen principalmente tres tipos de métodos para la evaluación del valor de los alimentos, que son los químicos, los enzimáticos y los biológicos [12]. La composición química de los alimentos permite realizar una caracterización y predecir el contenido en materiales digestibles, indicador fundamental del valor nutritivo [13, 14]. Los métodos de laboratorio se basan en reacciones químicas, pero los principales, desde el punto de vista práctico, son: Proteína bruta (PB), digestibilidad de la materia seca (DMS), y consumo animal voluntario de materia seca (CMS) [8]. El análisis químico provee información sobre algunos constituyentes químicos que afectan la digestión, (Van Soest, 1994), citado por [15]. Este análisis es la manera más simple para evaluar el alimento, llamado el análisis proximal o procedimiento Weende, divide el alimento en seis fracciones: Humedad, cenizas, proteína cruda, extracto etéreo, fibra cruda y extractos libres de nitrógeno $[15,16]$. Los factores que determinan el valor nutricional de un alimento son múltiples, sin embargo, deben considerarse al menos, la composición nutritiva, el consumo y la digestibilidad [17].

La calidad estará determinada por la digestibilidad [18] y el contenido en materiales digestibles es el indicador 
fundamental del valor nutritivo [13]. La digestibilidad de la materia seca (D.M.S.) estima la proporción del forraje consumido que es retenida en el cuerpo del animal [8]. Por tanto, el consumo y la digestibilidad son parámetros clave en cualquier sistema de evaluación de alimentos [18], y eficiencia energética [14]. Puede estimarse mediante métodos directos e indirectos. Entre los métodos directos se incluyen: Digestibilidad 'in vivo' (en el animal): Se mide la cantidad de materia seca consumida por 10-15 días de ensayo, y el volumen de heces producido. La diferencia entre ambas mediciones, llevada a porcentaje, da la digestibilidad aparente del forraje [8, 14]. Las mediciones in vivo proporcionan una medición estándar de la digestibilidad ya que representan la respuesta del animal a la dieta actual [18]

La digestibilidad de la materia orgánica determina el valor energético del forraje [14]. El valor energético se determina mediante ensayos de digestibilidad con animales y sólo pueden llevarse a cabo en determinados centros de investigación [19]. A la evaluación del valor de la energía todavía se considera es el factor más importante para determinar el costo del precio de la ración [20]. La cantidad de energía disponible se estima a partir de ecuaciones (McDonald et al., 1979), citado por [14]. La Energía metabolizable, corresponde a una manera de medir el contenido de energía nutricionalmente útil de un alimento [17]. La relación entre la energía neta y la energía metabolizable de un alimento representa la eficiencia $(k)$ con la cual la EM es utilizada para mantenimiento y propósitos productivos, siendo (1-k) la proporción de EM perdida por extra-calor [14]. Es por esta razón que como objetivos del proyecto está el reporte del análisis proximal, paredes celulares además de los coeficientes de digestibilidad y el valor de la energía útil (energía Metabolizable, energía para producción de leche y energía para ganancia de peso el NDT y la calidad de la dieta (q) de henos de alfalfa cosechados en las provincias de Chimborazo y Tungurahua.

\section{Materiales y Métodos}

La presente investigación se desarrolló en el Laboratorio de Nutrición Animal y Bromatología en la Facultad de Ciencias Pecuarias de la Escuela Superior Politécnica de Chimborazo ubicada en la Panamericana Sur, km 1 1/2 de la cuidad de Riobamba,

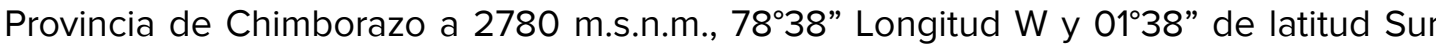
con una temperatura promedio de $13,8^{\circ} \mathrm{C}$; humedad relativa de $48,8 \%$ y precipitaciones de $432 \mathrm{~mm} / \mathrm{año}$.

Dicha investigación se realizó en cuatro fases, primero la evaluación nutricional mediante el análisis proximal, posteriormente la determinación de paredes celulares según el esquema de Van Soest, la determinación de los coeficientes de digestibilidad y por último la estimación de los valores de energía útil para los bovinos (energía Metabolizable [E.M.], energía neta de lactancia [E.N.I.], energía neta para ganado de carne [E.N.g.], el valor de los nutrientes digestibles totales [N.D.T.] y la calidad de la dieta [q]).

Las dietas utilizadas en este experimento, se adquirió pacas de henos elaborados en 5 diferentes regiones, dos de la Provincia de Tungurahua (Parroquia de Cunchibamba 
y Totoras y 3 de la provincia de Chimborazo (parroquias de san Juan, san Andrés y san Gerardo) y se codificó a las mismas como M1, M2, M3, M4 y M5.

De las dietas adquiridas una vez ingresadas se realizó el muestreo respectivo y se enviaron al laboratorio para el análisis proximal y paredes celulares. Las determinaciones bromatológicas de materia seca (MS), cenizas (C), extracto etéreo (EE), proteína cruda (PC) y fibra cruda (FC) se ejecutaron de acuerdo con los métodos oficiales de análisis según la [21]. Mientras que la fibra detergente neutra (FDN), fibra detergente acida (FDA) y lignina detergente acida (LDA) se determinaron según lo descrito en la técnica propuesta por Van Soest [22].Para determinar los coeficientes de digestibilidad se utilizaron 6 ovinos de un peso promedio de $30 \mathrm{~kg}$ los mismos que fueron ubicados en jaulas metabólicas siguiendo la metodología para la determinación del valor nutritivo de los forrajes tropicales de la Estación Experimental de Pastos y Forrajes 'Indio Hatuey', Matanzas, Cuba [23]. Después de un período de adaptación de los animales a las jaulas metabólicas y al alimento El heno de alfalfa se suministró en dos raciones en un nivel de consumo para mantenimiento más sal común y premezcla mineral, por un período de 11 días; las heces se recolecto una vez por día y por animal. Una vez registrado el peso se y se puso en congelación todas las muestras y se los conservó a $-10^{\circ} \mathrm{C}$. De temperatura hasta el final del experimento, luego de los cual se procedió a descongelar por $4 \mathrm{hr}$ previo al análisis respectivo.

Para la estimación de los valores de energía útil de los tratamientos (E.M., E.NI., E.Ng. N.D.T. y q) se utilizó las ecuaciones

realizadas por Van Es, A.J.H (1977).

\section{Resultados y Discusión}

1. Proteína: La Tabla 1 se aprecia el aporte nutricional en función de los contenidos de humedad, cenizas, proteína, fibra, extracto etéreo y Extracto libre de nitrógeno de 5 henos de alfalfa cosechados y henificados de diferentes lugares de la sierra centro del Ecuador, como lo son San Juan, San Gerardo, San Andrés, Cunchibamba y Totoras, en donde se aprecia que el mayor contenido de proteína corresponde al Heno de alfalfa procedente de la Parroquia totoras con un $21,37 \%$, según lo citado por Dammer, 2004 quien menciona que la alfalfa por ser una leguminosa de gran valor nutritivo posee alrededor de $24 \%$ de proteína en las hojas, valor expresado en Materia Seca (MS) y cerca del $10 \%$ de proteína en los tallos) valores que en promedio coinciden con lo citado tomando en cuenta que los análisis se trabajaron analizando la totalidad de la muestra, tanto hojas como tallos. Siendo en la alfalfa su alto contenido de proteína una de las características más representativas. El nivel de energía es medio a bajo y depende del estado de desarrollo en que es cosechada [17].

2. Humedad: Durante el balance de una ración, es fundamental conocer el contenido de agua en cada uno de los elementos que la componen; ya que niveles superiores al $8 \%$ favorecen la presencia de insectos y sobre el $14 \%$, existe el riesgo de contaminación por hongos y bacterias (Cockerell, 1971). En la muestra 
5, procedente de totoras en relación a las demás presentadas se apre cia un mayor porcentaje de humedad, incrementando así probabilidad contaminación microbiana, por otro lado, la muestra 2 procedente de San Gerardo presenta un menor contenido de humedad los cual resultaría más beneficiosa en cuanto a estabilidad de las muestras analizadas.

3. Cenizas: Mieres J. (2008) explica que la ceniza se considera como el contenido de minerales totales o material inorgánico en la muestra, es así que la muestra 4 procedente de cunchipamba presenta los valores más bajos expresados en base seca para éste parámetro, mientras que el resto de muestras de diferentes procedencias muestras valores más elevados pudiendo deberse principalmente a las características taxonómicas de las especies, Han y Baik [22] que depende directamente a la presencia de minerales en el suelo y su capacidad de absorción de nutrientes o su ciclo del cultivo.

4. Fibra cruda: Parámetro de gran interés en la alimentación animal es así que la alfalfa de la muestra M3, M4, procedentes de San Andrés y Cunchipamba presentan valores más elevados en cuanto a fibra, considerando que ésta estimula la masticación y a su vez la salivación, aumentando así la cantidad de bicarbonato que llega al rumen a través de la saliva, se control ade mejor manera e I pH ruminal evitando problemas de acidosis en los animales (Morales, 2013).

5. Extracto etéreo: La Muestra M5 procedente de la parroquia Totoras presenta un valor más elevado en este parámetro nutricional, considerado de gran importancia debido a que existe un mayor aporte de ácidos grasos, lípidos, vitaminas liposolubles y pigmentos vegetales, compuestos netamente energéticos relación a las otras muestras como los carotenoides. Algunos arbustos forrajeros como Leucaena leucocephala presentan contenidos superiores (4,6-6\%) (Llamas 2001). Estos valores se pueden ver influenciados también por las variaciones en la edad de las plantas al momento del análisis.

6. Extracto libre de nitrógeno: El análisis de la alfalfa muestra la presencia de una fracción nitrogenada, responsable de la capacidad de rebrote y variable según el estado vegetativo de la planta; podemos comprobar que esta fracción decrece conforme la planta alcanza la madurez, llegando a porcentajes entre 42 a 30\%, que no experimentan las variaciones detectadas en los demás parámetros. Bettini (1970) en este sentido podemos mencionar que la M3 corresponderían a un tipo de alfalfa en mayor estado de madurez perdiendo notablemente la presencia de nitrógeno en su composición.

7. Paredes celulares: En la Tabla 2 podemos observar durante los últimos años, los nutricionistas del ganado han empleado la fibra detergente neutra (FDN), la fibra detergente ácida (FDA) y la lignina detergente ácida (LDA) como indicadores de la energía dietética y de la ingesta, especialmente para las raciones de los rumiantes (eBook, 2018).

El consumo de heno varía de un $11 \%$ al $41 \%$, debido a factores como el clima, la edad del heno, según (Mendoza \& Morales, 2014) la FND disminuye cuando el porcentaje de 
nitrógeno aumenta lo cual se puede corroborar con los valores de proteínaobtenidos para la muestra 5 procedentes de la parroquia Totoras la cual presenta un menor contenido de FDN y un mayor porcentaje de proteína, es un valor muy importante en los rumiantes que consta de celulosa, hemicelulosa, lignina, además de residuos de nitrógeno y minerales.

Blanco (2005) mostró valores de FDA en el maní forrajero de 37,8\% y el centrosoma $(36,9 \%)$, y hace referencia a que contenidos elevados de FDA en los alimentos para animales puede ocasionar el llenado más rápido afectando la capacidad de consumo, así como también puede afectar la digestibilidad de otros componentes.es así que la muestra 3 procedente de san Andrés presenta u valor elevado de éste parámetro lo cual puede afectar de manera negativa a la alimentación.

Boudet (2000) menciona que las leguminosas herbáceas frecuentemente presentan un contenido de lignina alto, sosteniéndose que el mayor contenido de esta fracción se encuentra en los tallos que son el soporte mecánico de los órganos de las plantas y además influenciando en forma directa la conducción de agua y minerales por medio de transportadores lignificados, lo cual además se convierte en una limitante para el parámetro de digestibilidad, lo cual se puede apreciar en la muestra 3 procedente de San Andrés.

\section{Table 1}

Composición proximal de heno de alfalfa de diferentes parroquias de las provincias de Chimborazo y Tungurahua.

\begin{tabular}{|c|c|c|c|c|c|c|}
\hline Clase de muestra & $\begin{array}{l}\text { Humedad } \\
\text { total \% }\end{array}$ & $\begin{array}{l}\text { Cenizas B.S. } \\
\%\end{array}$ & $\begin{array}{l}\text { Proteína } \\
\text { cruda B.S. \% }\end{array}$ & $\begin{array}{l}\text { Fibra cruda } \\
\text { B.S. } \%\end{array}$ & $\begin{array}{l}\text { Extracto } \\
\text { etéreo B.S. } \\
\%\end{array}$ & $\begin{array}{l}\text { E.L.N. B.S. } \\
\%\end{array}$ \\
\hline $\begin{array}{l}\text { Heno de alfalfa DE San } \\
\text { Juan Rp-0019 }\end{array}$ & 8,85 & 9,35 & 18,82 & 36,60 & 1,91 & 33,45 \\
\hline Desviación estándar & 0,06 & 0,12 & 0,12 & 0,27 & 0,02 & 0,46 \\
\hline $\begin{array}{l}\text { Heno de alfalfa San } \\
\text { Gerardo Rp-00224 }\end{array}$ & 7,79 & 10,42 & 18,78 & 36,24 & 1,81 & 32,65 \\
\hline Desviación estándar & 0,06 & 0,49 & 0,31 & 0,05 & 0,02 & 0,41 \\
\hline $\begin{array}{l}\text { Heno de mezcla de } \\
\text { forrajes poca alfalfa } \\
\text { San Andrés Rp-00336 }\end{array}$ & 8,75 & 10,39 & 18,73 & 41,23 & 1,36 & 28,30 \\
\hline Desviación estándar & 0,04 & 0,02 & 0,10 & 0,21 & 0,22 & 0,09 \\
\hline $\begin{array}{l}\text { Heno de alfalfa } \\
\text { Cunchibamba } \\
\text { Rp-00521 }\end{array}$ & 9,73 & 9,16 & 18,74 & 41,69 & 2,06 & 28,55 \\
\hline Desviación estándar & 0,25 & 0,14 & 0,22 & 0,11 & 0,62 & 0,70 \\
\hline $\begin{array}{l}\text { Heno de alfalfa } \\
\text { Parroquia totoras } \\
\text { Rp-00535 }\end{array}$ & 9,99 & 10,50 & 21,37 & 31,64 & 2,13 & 34,46 \\
\hline Desviación estándar & 0,08 & 0,01 & 0,52 & 0,26 & 0,50 & 0,24 \\
\hline
\end{tabular}

B.S.: Base seca; E.L.N.: Extracto libre de nitrógeno.

Los resultados de la Tabla 3, en donde se observa los coeficientes de digestibilidad de los constituyentes del análisis proximal, los resultados comprueban las diferencias ( $p$ 
Table 2

Determinación de paredes celulares de heno de alfalfa de diferentes parroquias de las provincias de Chimborazo y Tungurahua.

\begin{tabular}{|c|c|c|c|}
\hline Clase de muestra & F.D.N. B.S. \% & F.D.A. B.S. \% & L.D.A. B.S. \% \\
\hline \multirow{2}{*}{$\begin{array}{l}\text { Heno de alfalfa DE } \\
\text { San Juan Rp-0019 }\end{array}$} & 45,59 & 41,75 & 8,51 \\
\hline & 0,40 & 0,12 & 0,18 \\
\hline $\begin{array}{l}\text { Heno de alfalfa San } \\
\text { Gerardo Rp-00224 }\end{array}$ & 47,22 & 40,84 & 8,38 \\
\hline Desviación estándar & 0,49 & 0,40 & 0,65 \\
\hline $\begin{array}{l}\text { Heno de mezcla de } \\
\text { forrajes poca alfalfa } \\
\text { San Andrés Rp-00336 }\end{array}$ & 51,77 & 45,47 & 9,30 \\
\hline Desviación estándar & 0,01 & 1,04 & 0,39 \\
\hline $\begin{array}{l}\text { Heno de alfalfa } \\
\text { Cunchibamba } \\
\text { Rp-00521 }\end{array}$ & 49,45 & 43,71 & 8,90 \\
\hline Desviación estándar & 0,22 & 0,02 & 0,75 \\
\hline $\begin{array}{l}\text { Heno de alfalfa } \\
\text { Parroquia totoras } \\
\text { Rp-00535 }\end{array}$ & 41,39 & 37,36 & 7,85 \\
\hline Desviación estándar & 1,32 & 0,26 & 0,48 \\
\hline
\end{tabular}

B.S.: Base seca; F.D.N.: Fibra detergente neutra; F.D.A.: Fibra detergente ácida; L.D.A.: Lignina detergente ácida.

$<0,0001)$, siendo los valores más altos para la digestibilidad de la materia seca, materia orgánica, proteína y el E.L.N. de 66,42\%,68,24\%, 80,23\% y 81,96\%, respectivamente, para el heno de alfalfa proveniente de la zona de San Juan y ubicándose en el.

Al respecto [24], en la tabla Holandesa de composición de los alimentos, encontraron valores de $63 \%$ a $69 \%$ como coeficiente de digestibilidad de la materia orgánica y entre $56 \%$ a $72 \%$ para la proteína cruda.

La digestibilidad de la materia orgánica de una planta forrajera depende esencialmente de su contenido en constituyentes de la pared celular y de su digestibilidad, disminuyendo ésta a medida que aumenta el contenido en paredes celulares y su grado de lignificación [19].

Por su parte Demarquilly y Andrieu (1992), citado por [19] indican un coeficiente de digestibilidad de la materia orgánica de entre $56,6 \%$ a $80,9 \%$.

El principal factor que causa el descenso de la digestibilidad de la materia orgánica con la madurez es la lignificación de las paredes celulares [19].

Borrajo [25] en un estudio realizado para determinar el consumo, rendimiento y digestibilidad del heno de alfalfa desde un estado de emergencia floral hasta un $100 \%$ de floración encontró para la digestibilidad de la materia seca un coeficiente de entre $55 \%$ a $65,9 \%$ y para la proteína cruda entre 62,9 a $70 \%$. Además Bruno et al. citado por [25], encontraron valores de entre $59,6 \%$ a $75,5 \%$ coeficientes de digestibilidad de la materia seca. 
Efecto del estado de madurez sobre la digestibilidad del forraje de alfalfa desde prebotón floral hasta el principio de la Semillación valores menores al 53\% y mayores al $65 \%$ [8].

A partir de los resultados de las evaluaciones, y una vez completadas las series analíticas que se describen a continuación, se determinó la digestibilidad aparente de la materia seca (DMS), materia orgánica (DMO) y energía (DE) [19]. Es sabido que a medida que la planta se acerca a la madurez se producen varios cambios típicos, uno de ellos es un aumento paulatino de la cantidad y grado de lignificación de las paredes celulares, con lo que disminuye su digestibilidad [11].

La Tabla 4 se recoge el aporte de energía metabolizable (E.M.), energía neta de la lactancia (E.NI.), energía neta de gananci a de peso (E.Ng), nutrientes digeribles totales (N.D.T.) y la calidad de la dieta (q) de 5 henos de alfalfa cosechados y henificad os de diferentes zonas de la región central del Ecuador, en la que se aprecia la diferencia estadísticamente significativa $(p<0,0001)$ que se produjo entre los diferentes henos de forraje de alfalfa de las diferentes zonas de la provincias de Chimborazo y Tungurahua. Al respecto el heno cosechado en la parroquia San Juan de la Provincia de Chimborazo, tuvo valores más altos en todos sus parámetros (E.M. $=2228 \mathrm{kcal} / \mathrm{kg}$ M.S., E.N.I. $=1245 \mathrm{kcal} / \mathrm{kg}$ M.S., E.N.g. $=1242 \mathrm{kcal} / \mathrm{kg}$ M.S., N.D.T. $=63,12 \%$ y q $=50,17 \%)$ con diferencias estadísticamente significativas $(p<0,0001)$ con los henos cosechados en las otras localidades, encontrándose los valores inferiores del heno de alfalfa en la zona de Cunchibamba, provincia de Tungurahua, (E.M. $=2000 \mathrm{kcal} / \mathrm{kg}$ M.S., E.N.I. = $1091 \mathrm{kcal} / \mathrm{kg}$ M.S., E.N.g. $=1039 \mathrm{kcal} / \mathrm{kg}$ M.S., N.D.T. $=57,05 \%$ y $q=44,39 \%)(p<0,0001)$. Se observa que los henos de alfalfa de las parroquias de San Gerardo, San Andrés de la provincia del Chimborazo y parroquia Totoras de la provincia de Tungurahua demostraron valores intermedios de las zonas de San Juan Provincia de Chimborazo y Cunchibamba provincia de Tungurahua en donde no se observaron variaciones estadísticamente significativas $(p<0,0001$ ). Holland y Kezar en un estudio realizado del heno de alfalfa cortada en diferentes estados fenológicos en cuanto al N.D.T. encontró valores desde el $55 \%$ al $66 \%$ de la materia seca desde el material de flor tardía hasta en vegetación tardía respectivamente [8]. Del valor nutricional de la harina de alfalfa estudiado por [26], encontró valores de $56 \%$ para el N.D.T. y 2,08 Mcal $/ \mathrm{kg}$. De la misma manera [27], en cuatro experimentos realizados con un monocultivo de alfalfa, en realizó una comparación de esta forrajera sin lluvia, con lluvia artificial y lluvia natural, entre otros componentes del val or nutritivo a N.D.T. en \%, habiendo encontrado un rango de diferencia entre $49,6 \%$ hasta $62,9 \%$ entre los cuatro diferentes experimentos. Blok y Spek (2016) logran el valor de la energía neta de lactancia para bovinos está en un rango entre 1008 y $1898 \mathrm{kcal} / \mathrm{kg}$ de la materia seca y la energía neta para ganancia de peso en bovinos de carne está entre valores de 995 a $2026 \mathrm{kcal} / \mathrm{kg}$ de la materia seca. Por otro lado [28], reportan valores de un 50 al 72\% para el N.D.T., 1780 hasta 2530 $\mathrm{kcal} / \mathrm{kg}$ de M.S. para la E.M., de entre 1209 a $1790 \mathrm{kcal} / \mathrm{kg}$ de M.S. para la E.N.I. y 1640 a $1823 \mathrm{kcal} / \mathrm{kg}$ de M.S. para la E.N.g. Al realizar el análisis comparativo de las diferentes variables del presente estudio con la información obtenida de autores todos los datos están dentro de los rangos observados, pero que la diferencia estadística significativa entre los diferentes tratam ientos, dependerá del estado fenológico o de madurez de 


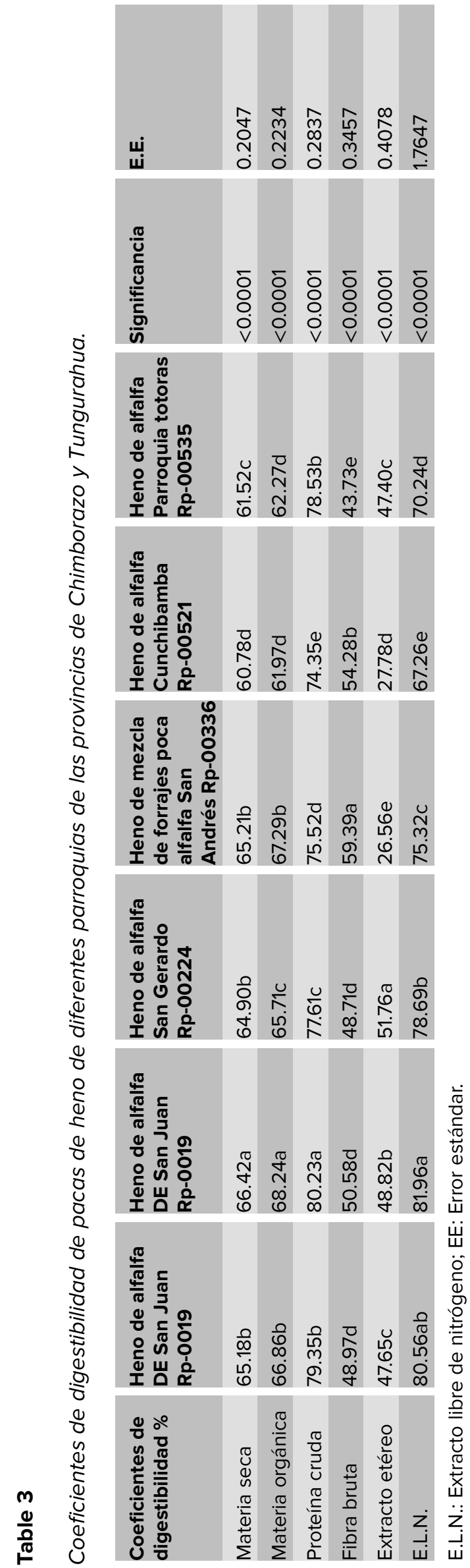




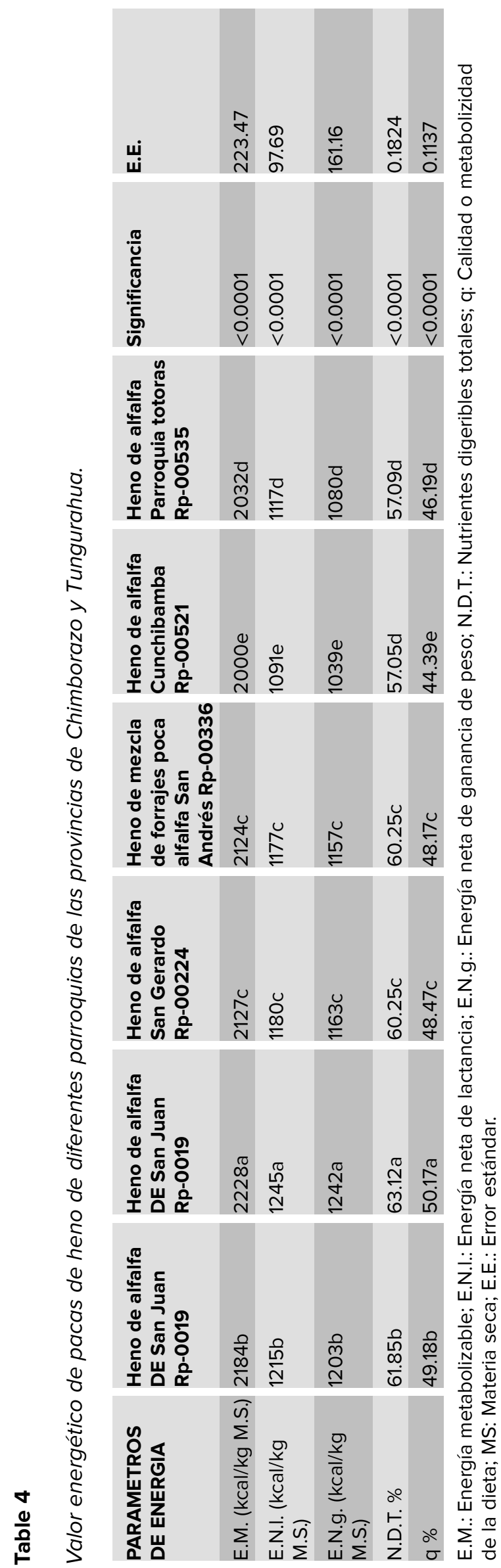


la planta, que es el factor más importante a tener en cuenta en el momento del corte para producir forraje conservado de alta calidad, ya que expresa el efecto acumulado que el medio ambiente y el genotip o tienen sobre la planta [8], o que la calidad del heno de alfalfa (valor nutritivo) podría verse influenciada por factores como factores climáticos, factores edáficos como las condiciones del suelo, pérdida de hojas durante la producción d e heno, prácticas de almacenamiento y alimentación, enfermedades e insectos, malezas, cultivo de alfalfa, humedad contenido durante el almacenamiento. Destacó que el valor nutritivo de los forrajes puede variar considerablemente [29]. Por otro lado [17], al realizar un estudio de la alfalfa en distintos estados fenológicos encontró valores de energía metabolizable de entre 1,83 a 2,72 Mcal/kg M.S., e indica que su principal valor nutricional es su alto contenido de proteína. El nivel de energía es medio a bajo y depende del estado de desarrollo en que es cosechada.

\section{Conclusiones}

Del análisis proximal de los cincos henos de alfalfa de diferentes lugares de la sierra centro del Ecuador, como lo son San Juan, San Gerardo, San Andrés, Cunchibamba y Totoras, se encontró el mayor contenido de proteína en el heno de alfalfa procedente de la Parroquia Totoras con un $21,37 \%$, un menor porcentaje de humedad en el heno procedente de San Gerardo con 7,79\% y el mayor porcentaje de humedad el heno procedente de Totoras con 9,99\%; las cenizas de la muestra de heno de Totoras presentó el valor más alto con 10,50\%, mientras que el heno de Cunchipamba presentó el valores más bajo con el 9,16\%; en lo referente a la fibra bruta se determinó el valor más alto con el heno de alfalfa de la muestra de Cuchibamba con $41,69 \%$ y con el valor más bajo el heno de alfalfa de la parroquia de Totoras con 31,64\%; el extracto etéreo reportó que la muestra procedente de la parroquia Totoras presenta el valor más elevado con 2,13\%; en cuanto al extracto libre de nitrógeno el heno procedente de la parroquia de Totoras $34,46 \%$ posee al porcentaje más alto.

El heno de la parroquia Totoras presentó un menor contenido de FDN con 41,39\% y un mayor porcentaje de proteína, por otra parte la muestra procedente de San Andrés presentó un valor de 51,77\% que es elevado lo cual puede afectar de manera negativa a la alimentación animal.

Los coeficientes de digestibilidad del heno de alfalfa proveniente de la zona de San Juan para materia seca, materia orgánica, proteína y el E.L.N. de 66,42\%, 68,24\%, $80,23 \%$ y $81,96 \%$, respectivamente son más elevados que el resto de muestras analizadas.

La energía metabolizable (E.M.), energía neta de la lactancia (E.N.I.), energía neta de ganancia de peso (E.N.g.), nutrientes digeribles totales (N.D.T.) y la calidad de la dieta (q) de los 5 henos de los henos de la parroquia San Juan de la Provincia de Chimborazo, tuvo valores más altos en todos sus parámetros (E.M. $=2228 \mathrm{kcal} / \mathrm{kg}$ M.S., E.N.I. $=1245$ $\mathrm{kcal} / \mathrm{kg}$ M.S., E.N.g. $=1242 \mathrm{kcal} / \mathrm{kg}$ M.S., N.D.T. $=63,12 \%$ y q $=50,17 \%)$ a diferencia de los henos cosechados en las otras localidades. 
Se encontraron valores inferiores del heno de alfalfa en la zona de Cunchibamba, provincia de Tungurahua, (E.M. $=2000 \mathrm{kcal} / \mathrm{kg}$ M.S., E.NI. $=1091 \mathrm{kcal} / \mathrm{kg} \mathrm{M.S.,} \mathrm{E.N.g.} \mathrm{=}$ $1039 \mathrm{kcal} / \mathrm{kg}$ M.S., N.D.T. $=57,05 \%$ y q $=44,39 \%)$.

Los henos de alfalfa de las parroquias de San Gerardo, San Andrés de la provincia del Chimborazo y parroquia Totoras de la provincia de Tungurahua demostraron valores intermedios de las zonas de San Juan Provincia de Chimborazo y Cunchibamba.

\section{References}

[1] Haro R. Informe sobre recursos zoogenèticos Ecuador. Ministerio De Agricultura Y Ganaderia; 2003.

[2] Caparo C. Características físicas y socioeconómicas del país 1.1. Capítulo. 2003;1(1):88.

[3] Requelme N, Bonifaz N. Caracterización de sistemas de producción lechera de Ecuador. La Granja. 2012;15(1):55.

[4] INEC. Encuesta de superficie y producción agropecuaria continua. Instituto Nacional de Estadísticas y Censos; 2014.

[5] Clavijo E, Cadena P. (2011). Statewide Agricultural Land Use Baseline 2015 (Vol. 1). Producción y calidad nutricional de la alfalfa (medicago sativa) sembrada en dos ambientes diferentes y cosechada en distintos estadios fenológicos.

[6] Petisco C, Criado BG, Criado LG, Aldana BRVDE. Aplicación de la técnica nirs para el análisis de materia seca y proteína en hojas de alfalfa. 2004.

[7] Guerrero-rodríguez J. Growth and nutritive value of lucerne (Medicago sativa L.) and Melilotus (Melilotus albus Medik.) under saline conditions. 2006;May:158.

[8] Juan NA, Romero NA, Bruno OA. Conservacion del forraje de alfalfa. La Alfalfa En La Argentina. 1995;70:173-194.

[9] Michelena JB. Conservación del forraje en el trópico. 1995.

[10] Fari T. Guía de suplementación alimenticia estratégica para bovinos en época seca. 2008. Vásquez M. Escuela politécnica nacional. 2010.

[11] Ribeiro JMCR, Moreira OMSC. in the Project. 1998;91:79-91.

[12] Gasa J, Castrillo C. Criterios de utilizacion de subproductos agroindustriales en la alimentacion de rumiantes. Hojas Divulgadoras, Núm. 1991;13(91):24.

[13] Gonzalez-Arraez A, Castro P. Factores que afectan a la calidad del ensilaje de hierba y a la planta de maíz forrajero en Galicia y evaluación de métodos de laboratorio para la predicción de la digestibilidad in vivo de la materia orgánica de estos forrajes ensilados. Journal of Petrology. 2013;369(1):1689-1699.

[14] Muro A. Efectos de la fuente de fibra detergente neutro, fibra detergente ácido y proteína sobre la cinética de degradación ruminal in vitro. 2007.

[15] Maiztegui J. Composicion Analisisy clasificaciondelos Alimentos. 2010.

[16] Bravo J. Caracterización nutricional de forrajes verdes, forrajes secosa, concentrados y subproductos agroindustriales para la alimentación del ganado en la zona sur. In Vitro. 2008;3;1-174.

[17] Naranjo JF. Caracterización nutricional y de la cinética de degradación ruminal dealgunos de los recursos forrajeros con potencial para la suplementaciónde rumiantes en el trópico alto de Colombia. Ces Med. Vet. Zootec. 2011;6(1):9-19.

[18] Gonzalez-Arraez FGCP. Predicción de la digestibilidad in vivo de la materia orgánica de ensilajes de hierba y maíz por métodos de laboratorio. Fedna. 2003;111-132.

[19] De Boever J, Guevara P, Cottyn B, De Brabander D, Vanacker J, Boucqué C. Ecuaciones para predecir los valores de digestibilidad y energía de ensilajes de gramíneas, ensilajes de maíz, henos de gramíneas, piensos compuestos y materias primas para ganado. (No. 991). 2000.

[20] AOAC. Modification to AOAC official methods 2009.01 and 2011.25 to allow for minor overestimation of low molecular weight soluble dietary fiber in samples containing starch. Journal of AOAC International. 2014; 97(3):896901.

[21] Goering HK, Van Soest P. Forage fiber analyses. U.S. Department of Agriculture. 1975:379;387-598.

[22] González O, Cáceres E. Metodologia para la determinacion del valor nutritivo de los forrajes tropicales. 2015.

[23] Blok M.-C., Spek J.-W. CVB feed table 2016. 2016 August 631. Available from: www.cvbdiervoeding.nl

[24] Borrajo J. 1965. a7915E.Pdf. p. 94. 
[25] Huertas C, DW. Evaluación del uso de harina de alfalfa, harina de arroz y salvado de trigo, sobre los parámetros productivos en conejos de ceba en el municipio de Garacoa (Issue hal 140). Universidad Nacional Abierta y a distancia; 2014.

[26] Coblentz WK, Muck RE. Effects of natural and simulated rainfall on indicators of ensilability and nutritive value for wilting alfalfa forages sampled before preservation as silage. Journal of Dairy Science. 2012;95(11):6635-6653.

[27] Tables, U. S., Composition, F., Data, N., States, U., Feeds, C., Subcommitte, T. R., Composition, F., Nutrition, A., Isbn, C., Pdf, T., Press, N. A., Press, N. A., Academy, N., Academy, N., \& Press, N. A. United States - Canadian Tables of Feed Composition (N. R. C. Subcommitte on Feed Composition, Committee on Animal Nutrition). 1982.

[28] Scholtz GDJ, van der Merwe HJ, Tylutki TP. The nutritive value of South African Medicago sativa L. hay. South African Journal of Animal Sciences. 2009;39(Suppl. 1):79-182. 